> L'apparition de nucléotides dans les fluides extracellulaires résulte de la nécrose cellulaire, de l'exocytose de granules sécrétoires (vésicules synaptiques, granules denses des plaquettes) ou du flux sortant via des transporteurs membranaires. Les nucléotides extracellulaires sont transformés rapidement par des ecto-nucléotidases présentes de manière ubiquitaire à la surface des cellules. Deux familles de récepteurs sont responsables des effets des nucléotides extracellulaires : les récepteurs $\mathrm{P} 2 \mathrm{X}$ qui possèdent une activité intrinsèque de canal ionique et les récepteurs P2Y couplés aux protéines G. Actuellement, huit récepteurs P2Y humains authentiques ont été identifiés. L'importance physiologique et le potentiel pharmacothérapeutique de certains d'entre eux sont maintenant établis. Les récepteurs $P 2 y_{1}$ et $P 2 Y_{12}$ jouent un rôle crucial dans l'agrégation des plaquettes par l'ADP et le récepteur P2Y ${ }_{12}$ constitue la cible du clopidogrel, un agent antithrombotique majeur. Via le récepteur $\mathrm{P} 2 \mathrm{Y}_{2}$, les nucléotides stimulent la sécrétion épithéliale de chlorure et augmentent ainsi l'hydratation du mucus bronchique et la lubrification de la surface oculaire. Enfin, des résultats récents suggèrent que le récepteur $\mathrm{P} 2 \mathrm{Y}_{11}$ joue un rôle important dans l'hématopoï̀se et l'immunomodulation. <

\section{Les récepteurs P2Y des nucléotides extracellulaires: du clonage à la physiologie}

Jean-Marie Boeynaems, Didier Communi, Nathalie Suarez Gonzalez, Béatrice Hechler, Catherine Léon, Christian Gachet

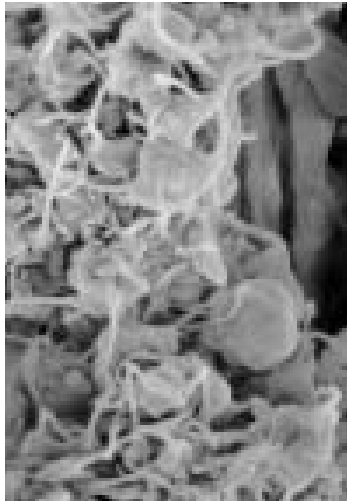

nyliques (ATP, ADP), puis pour les nucléotides uraciliques (UTP, UDP) et, récemment, pour une molécule plus inattendue, I'UDP-glucose. Les nucléotides extracellulaires exercent des effets dans tous les systèmes et peu de types cellulaires sont complètement dépourvus de récepteurs des nucléotides. Parmi ces multiples actions des nucléotides extracellulaires, celles qui sont réellement pertinentes d'un point de vue physiopathologique commencent à émerger, en particulier grâce à l'invalidation génique chez la souris.

leurs multiples fonctions intracellulaires, nucléotides exercent un rôle ubiquitaire en tant que médiateurs intercellulaires locaux [1]. Les trois événements impliqués dans ce rôle des nucléotides extracellulaires commencent à être bien caractérisés : libération dans les fluides extracellulaires, dégradation par les ectonucléotidases et action via des récepteurs membranaires. L'identification des récepteurs n'est pas terminée, mais elle a déjà conduit à étendre le champ des nucléotides impliqués dans la signalisation intercellulaire : des récepteurs ont en effet été identifiés pour les nucléotides adé-

\section{Libération des nucléotides dans les fluides extracellulaires}

L'apparition des nucléotides dans les fluides extracellulaires peut se faire par trois mécanismes : lyse cellulaire, exocytose de granules sécrétoires et flux sortant par des transporteurs membranaires (Figure 1). La libération de nucléotides par des cellules nécrotiques aboutit à une 
concentration suffisamment élevée dans le milieu pour activer les récepteurs des cellules du système immunitaire. L'exocytose des nucléotides intervient lors de la transmission synaptique (I'ATP est co-localisé dans les vésicules synaptiques avec des neurotransmetteurs plus conventionnels comme l'acétylcholine et la noradrénaline) et de l'agrégation plaquettaire (les granules denses des plaquettes concentrent I'ATP et l'ADP). Ainsi, lorsque les plaquettes sont stimulées par la thrombine, les concentrations d'ADP et d'ATP à la surface des plaquettes atteignent transitoirement des valeurs supérieures à 100 $\mu M$. Le flux sortant via des transporteurs membranaires est une réponse à différentes stimulations mécaniques : force de cisaillement exercée par le flux sanguin sur l'endothélium vasculaire, gonflement osmotique des cellules de l'épithélium ciliaire, étirement des cellules épithéliales urinaires lors de la distension de la vessie [2]. In vitro, un simple changement du milieu de culture suffit à induire la libération de $1,5 \%$ du contenu cellulaire en nucléotides, et à provoquer la stimulation autocrine de récepteurs tant par I'ATP que par I'UTP. Les concentrations atteintes dans ces conditions sont d'environ $70 \mathrm{nM}$ pour I'UTP et 200 nM pour l'ATP, ce qui reflète le rapport des concentrations intracellulaires. L'emploi de la microscopie à force atomique a révélé le caractère focal de ce flux sortant et a démontré que la concentration en nucléotides en certains sites de la surface membranaire est nettement supérieure à celle qui est atteinte dans le milieu ambiant [3]. L'identité des transporteurs impliqués reste controversée [4]: des résultats contradictoires ont été rapportés quant au rôle de protéines $\mathrm{ABC}$ (ATP binding cassette) comme le CFTR (cystic fibrosis transmembrane regulator) ou la glycoprotéine $P$ et le rôle d'hémiconnexines a également été proposé.

\section{Les ecto-nucléotidases}

Les nucléotides extracellulaires sont transformés rapidement par des enzymes présentes à la surface de nombreux types cellulaires et nommées ecto-nucléotidases. Ainsi,
I'ATP, lorsqu'il est perfusé dans la circulation pulmonaire, a une demi-vie inférieure à $l$ seconde. La famille des ectonucléotidases rassemble plusieurs membres dont la structure et la fonction sont bien caractérisées (Figure 1). II s'agit des ecto-nucléosides 5'-triphosphates diphosphohydrolases ( $\varepsilon$-NTPDase), qui hydrolysent les nucléosides 5'-triphosphates et/ou nucléosides 5'-diphosphates en nucléosides 5'-monophosphates. Cette famille compte six sous-types (CD39, CD39L1-L5), renommés récemment NTPDase 1-6 [5], qui se distinguent par l'hydrolyse préférentielle ou exclusive de nucléosides 5'-triphosphates versus celle des 5'-diphosphates. Leur nom dérive du CD39, un marqueur de différenciation des lymphocytes cloné en 1994 et dont l'activité ATP-diphosphohydrolase avait été identifiée plus tard. Ces NTPDases présentent un seul ou deux domaines transmembranaires avec des extrémités $N$ et $\mathrm{C}$-terminales intracellulaires. Elles existent sous forme d'homo-oligomères (dimères, trimères ou tétramères). Ces enzymes sont présentes dans de nombreux tissus et notamment sur les cellules endothéliales vasculaires où elles inhibent l'interaction des plaquettes avec la paroi vasculaire, en dégradant l'ADP, médiateur majeur de l'activation plaquettaire [6]. Elles ont également un rôle modulateur de l'activation des cellules endothéliales. Des espoirs ont

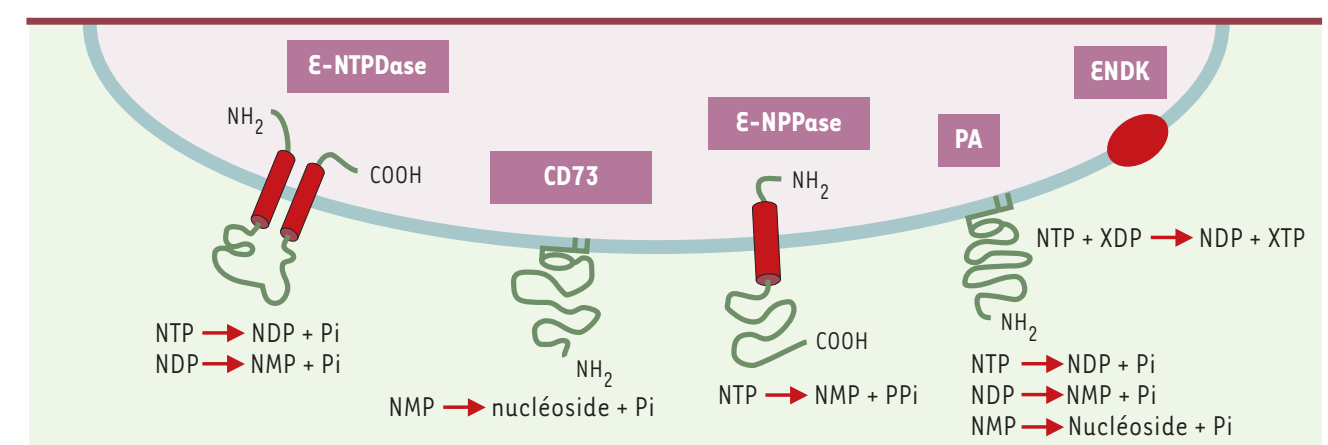

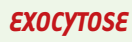

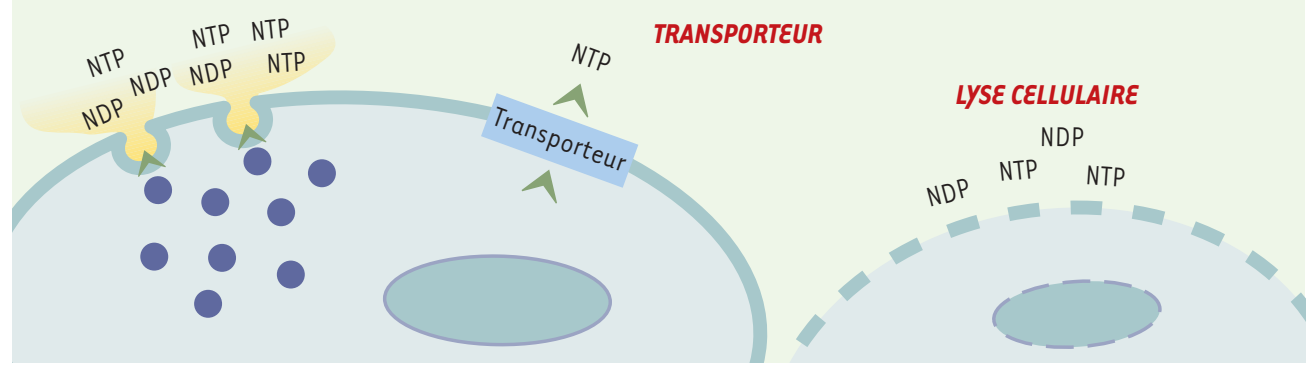

Figure 1. Source des nucléotides extracellulaires et métabolisme par les ecto-enzymes. Les nucléotides libérés soit par des transporteurs spécifiques, soit par exocytose, ou encore lors de la nécrose des cellules, peuvent être métabolisés par les ecto-enzymes présentes à la surface des cellules. PA : phosphatase alcaline ; CD73 : ecto5'-nucléotidase ; $\varepsilon$-NPPase : ecto-nucléotide pyrophosphatase/phosphodiestérase ; $\varepsilon$-NTPDase : ecto-nucléoside 5'-triphosphate diphosphohydrolase ; ENDK : ecto-nucléotide diphosphokinase (structure non représentée car encore inconnue) ; NDP (ou XDP) : nucléotide diphosphate; NMP : nucléotide monophosphate ; NTP (ou XTP) : nucléotide triphosphate; $\mathrm{Pi}$ : phosphate inorganique ; $\mathrm{PPi}$ : pyrophosphate. 
été fondés sur la production et l'utilisation comme agents antithrombotiques de formes solubles du CD39 et sur le transfert de gène pour la prévention des rejets d'allogreffes. La production de souris déficientes pour l'expression du CD39 (NTPDasel) a permis de confirmer l'importance de cette enzyme dans l'hémostase et dans la thrombose ainsi qu'en matière de transplantation [7]. Cependant, outre des dépôts de fibrine dans de multiples organes, ces souris présentent un phénotype paradoxal d'hypofonctionnement plaquettaire avec un allongement du temps de saignement et une résistance à la thrombose, dus à la désensibilisation du récepteur P2y ${ }_{1}$ [7].

Une deuxième catégorie d'enzymes, nommées ectonucléotide pyrophosphatases/phosphodiestérases ( $\varepsilon$ NPPase), assure la conversion de l'ATP en AMP et en pyrophosphate ainsi que l'hydrolyse de l'AMP-3', 5'-cyclique en AMP. Elles hydrolysent les nucléotides purinergiques et pyrimidinergiques. Six sous-types ont été clonés et rassemblés en trois groupes nommés NPPl-3. Ces enzymes présentent un seul domaine transmembranaire avec une extrémité C-terminale extracellulaire. Quant aux phosphatases alcalines ou ecto-phosphomonoestérases, elles libèrent du phosphate inorganique à partir de nucléosides 5'-tri-, di- et monophosphates. Elles sont ancrées dans la membrane plasmique par un groupement glycosylphosphatidylinositol (GPI). C'est également le cas de l'ecto-5'nucléotidase ou CD73, un marqueur de maturation des lymphocytes B et T. Cette enzyme catalyse l'étape finale d'hydrolyse des nucléosides 5'-monophosphates en nucléosides et Pi. Elle est essentielle à la formation d'adénosine extracellulaire. Enfin, d'autres enzymes à activité ecto-nucléotide diphosphokinase (ENDK) ont été caractérisées. Elles catalysent l'interconversion de nucléotides 5'di- et triphosphates : ainsi, I'UTP en présence d'ADP est converti en UDP et en ATP. Sur les plaquettes sanguines, une ENDK assure la conversion d'ADP en ATP.

L'existence de ces multiples enzymes ajoute un degré de complexité considérable dans la régulation des effets physiologiques des nucléotides au niveau cellulaire et tissulaire. Elle complique également la caractérisation pharmacologique des récepteurs des nucléotides.

\section{Les récepteurs des nucléotides}

Traditionnellement, les actions des nucléotides extracellulaires ont été attribuées à des récepteurs P2 ( $P$ pour purine) par opposition aux récepteurs $\mathrm{Pl}$ de l'adénosine. Jusqu'aux premiers clonages de récepteurs P2 qui ont été rapportés en 1993, la nomenclature pharmacologique et physiologique distinguait essentiellement les récepteurs P2X (effet vasoconstricteur de I'ATP), P2Y (effet vasodilatateur de l'ATP et de l'ADP, dépendant de l'endothélium), P2U (effet vasodilatateur de I'UTP), P2T (effet activateur de l'ADP sur les plaquettes sanguines) et P2Z (formation de pores non sélectifs dans les monocytes). Ces récepteurs étaient diversement distribués dans de nombreux types cellulaires et tissus comme les récepteurs P2X et P2Y ou alors restreints à un type cellulaire comme c'est le cas du récepteur P2T (T pour thrombocytes) que l'on pense spécifique de la lignée mégacaryocyto-plaquettaire. À partir de 1994, cette nomenclature pharmacophysiologique a été remplacée par une classification moléculaire fondée sur l'existence de deux familles : les récepteurs $P 2 X$ possédant une activité intrinsèque de canal ionique, et les récepteurs P2Y couplés aux protéines $G$. Dans chaque famille, les sous-types sont désignés par un indice attribué selon la chronologie des clonages. On en est ainsi arrivé à sept récepteurs $P 2 X$ et quatorze récepteurs $P 2 y$. Cependant, il est maintenant évident que plusieurs sous-types de récepteurs P2y ont été inclus à tort dans cette classification, sur la base d'un certain degré d'homologie de structure sans preuve fonctionnelle solide ou en ne tenant pas compte de la parenté phylogénétique. Ainsi, le récepteur $\mathrm{P}_{2} \mathrm{Y}_{3}$ est en fait l'orthologue aviaire du récepteur $\mathrm{P} 2 \mathrm{y}_{6}$, tandis que le récepteur $P 2 Y_{8}$ est l'orthologue de xénope $d u$

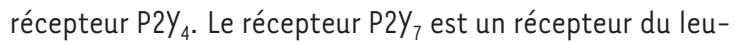

\begin{tabular}{|c|c|c|c|c|c|c|}
\hline Groupe & Sous-type & Chromosome & Agoniste & Protéine G & Localisation & Références \\
\hline \multirow[t]{5}{*}{ I } & $\mathrm{P}_{2} \mathrm{y}_{1}$ & $3 q 24-25$ & $A D P$ & $\mathrm{G}_{\mathrm{q}}$ & Étendue & [29] \\
\hline & $\mathrm{P} 2 \mathrm{y}_{2}$ & $11 q 13.5$ & ATP $=U T P$ & $G_{q}\left(+G_{i}\right)$ & Étendue & [30] \\
\hline & $\mathrm{P}_{2} \mathrm{y}_{4}$ & $\mathrm{Xq13}$ & UTP & $G_{q}\left(+G_{i}\right)$ & Placenta, poumon, oreille interne & [31] \\
\hline & $\mathrm{P}_{2} \mathrm{y}_{6}$ & $11 q 13.5$ & UDP & $\mathrm{G}_{\mathrm{q}}$ & Étendue & [32] \\
\hline & $\mathrm{P}_{2} \mathrm{y}_{11}$ & $19 p 31$ & ATP & $G_{q}+G_{s}$ & Rate, intestin, foie & [33] \\
\hline \multirow[t]{3}{*}{ II } & P2 $\mathrm{Y}_{12}$ & $3 q 21-25$ & ADP & $\mathrm{G}_{\mathrm{i}}$ & Plaquettes, cerveau & {$[34,35]$} \\
\hline & $\mathrm{P} 2 \mathrm{y}_{13}$ & $3 q 24-25$ & ADP & $\mathrm{G}_{\mathrm{i}}$ & Système immunitaire, cerveau & {$[36]$} \\
\hline & $\mathrm{P} 2 \mathrm{Y}_{14}$ & $3 q 24-25$ & UDP-glucose & $\mathrm{G}_{\mathrm{i}}$ & Étendue & {$[37]$} \\
\hline
\end{tabular}

Tableau 1. Classification des récepteurs P2Y humains. 
cotriène $B 4$, alors que les récepteurs $P 2 y_{5}, P 2 y_{9}$ et $P 2 y_{10}$ doivent être considérés comme des récepteurs orphelins. Certains des récepteurs P2y $\left(P 2 y_{4}, P 2 y_{6}\right)$ ont une forte sélectivité pour les nucléotides uraciliques, ce qui rend inadéquat le terme de récepteurs purinergiques qui doit être remplacé par récepteurs purinergiques et pyrimidinergiques ou récepteurs des nucléotides. Les récepteurs P2y sont couplés principalement à $G_{q}$ ou $G_{i}$ : jusqu'à présent, le seul membre de la famille $P 2 y$ couplé à $G_{s}$ est le récepteur $P 2 Y_{11}$. Plusieurs récepteurs $P 2 y$ induisent également l'activation de la voie Rho-Rho kinase.

Les premiers récepteurs $P 2 y$ clonés, $P 2 Y_{1}$ et $P 2 y_{2}$, corres- $(\rightarrow) \mathrm{m} / \mathrm{s}$ 2001, n⿳6-7, p. 787 pondaient aux récepteurs $\mathrm{P} 2 \mathrm{Y}$ et $\mathrm{P} 2 \mathrm{U}$ de la nomenclature pharmacologique et leur existence était donc attendue sur la base des données pharmacologiques disponibles.

L'identification des récepteurs $P 2 y_{4}, P 2 y_{6}$ et $P 2 y_{11}$, fruit d'une stratégie de clonage par homologie, était inattendue. Malgré d'intenses efforts, le clonage par homologie n'a pas permis d'isoler le récepteur plaquettaire de l'ADP couplé à $G \alpha_{i}$, appelé $P 2 T_{A C}, P 2 C Y C$ ou P2y ${ }_{A D P}$, dont l'existence était fondée sur de multiples données pharmacologiques. Lorsque ce récepteur a été identifié et baptisé $P 2 Y_{12}$, il est apparu que sa séquence présentait peu d'homologie avec les autres récepteurs P2y identifiés jusqu'alors. En revanche, il est structurellement apparenté à un récepteur de I'UDP-glucose, maintenant rebaptisé $P 2 Y_{14}$, et à un autre récepteur de I'ADP caractérisé dans la foulée, $P 2 Y_{13}$. Il est intéressant de noter que les gènes humains codant pour les récepteurs $P 2 y_{1}, P 2 y_{12}, P 2 y_{13}$ et $P 2 y_{14}$ sont localisés à proximité l'un de l'autre (région $3 q 24$ ), ce qui suggère l'existence d'un ancêtre commun. Il apparaît donc que la famille des récepteurs P2Y est constituée de deux groupes de récepteurs structurellement distincts. Cette situation est étrangement similaire au cas des récepteurs de l'histamine : là aussi l'isolement du récepteur $\mathrm{H}_{3}$ a été retardé par sa faible homologie avec les récepteurs $H_{1}$ et $H_{2}$ et a été rapidement suivi de l'identification du récepteur $\mathrm{H}_{4}$ [8]. Donc, à ce stade, huit récepteurs P2y humains authentiques ont été identifiés (Tableau 1, Figure 2), mais l'existence de plusieurs récepteurs orphelins structurellement apparentés suggère que le nombre final sera plus élevé. Enfin, il faut souligner que la découverte du récepteur $\mathrm{P} 2 \mathrm{Y}_{14}$ de I'UDP-glucose a considérablement étendu le champ des ligands des récepteurs P2y et indique un rôle inattendu de signalisation cellulaire pour ce type de molécules $(\rightarrow)$.

\section{Rôles physiologiques et potentiel pharmacothérapeutique}

L'importance physiopathologique et le potentiel pharmacothérapeutique de certains récepteurs $P 2 Y$ sont maintenant établis à des degrés divers.
- Les récepteurs $P 2 Y_{1}$ et $P 2 Y_{12}$ jouent un rôle crucial dans l'hémostase et la thrombose.

- Le récepteur $\mathrm{P} 2 \mathrm{Y}_{2}$ constitue une cible intéressante pour augmenter l'hydratation du mucus bronchique et la lubrification de la surface oculaire, via une sécrétion accrue de chlorure par les cellules épithéliales.

- Des résultats récents suggèrent l'intervention des récepteurs $\mathrm{P} 2 \mathrm{Y}$, en particulier le récepteur $\mathrm{P} 2 \mathrm{Y}_{11}$, dans l'hématopoïèse et le système immunitaire.

\section{Hémostase et thrombose}

Les nucléotides adényliques, et l'ADP en particulier, jouent un rôle capital dans l'activation et l'agrégation des plaquettes sanguines. Les plaquettes sont des fragments anucléés de mégacaryocytes dont le rôle majeur est d'assurer I'hémostase, c'est-à-dire l'arrêt du saignement dans les vaisseaux de petit calibre. Ces mêmes éléments sont impliqués dans les complications thrombo-emboliques ischémiques de l'athérosclérose ainsi que dans la resténose après angioplastie transluminale. En 1961, l'ADP a été le premier agent agrégant plaquettaire de petit poids moléculaire à être identifié [9]. Les nucléotides adényliques

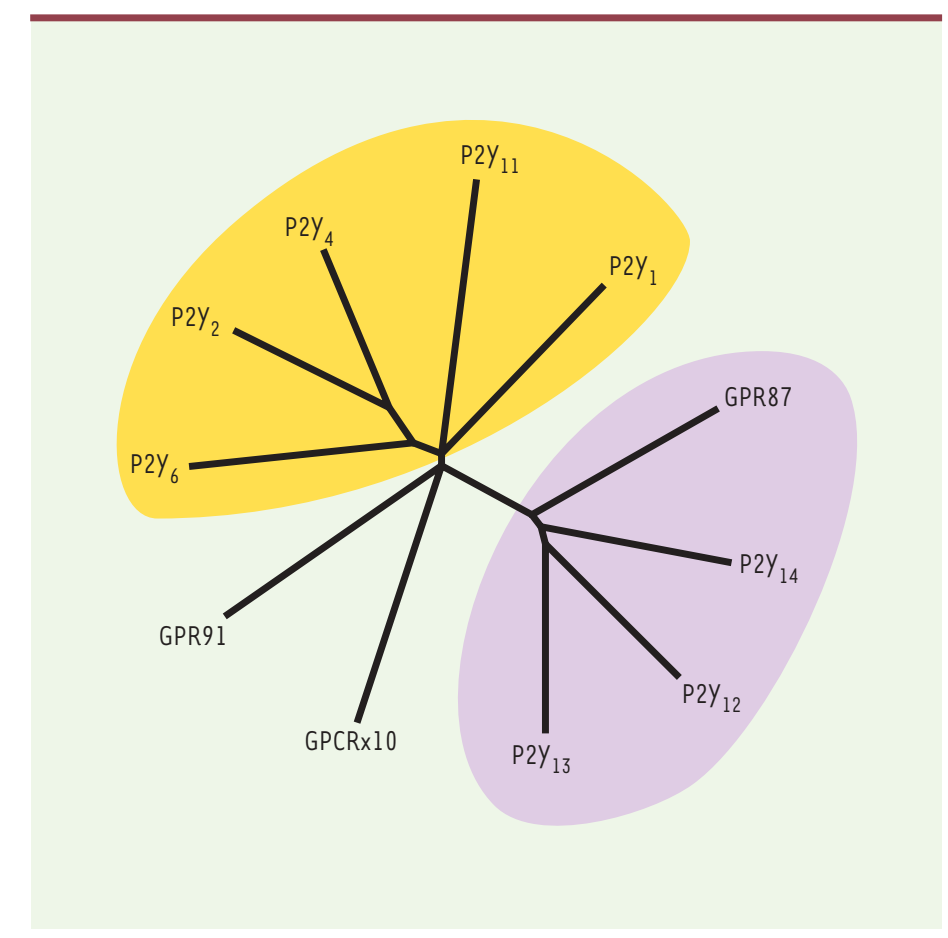

Figure 2. Arbre phylogénétique des récepteurs $P 2 y$. Cet arbre a été construit en utilisant le programme TreeView, après alignement des séquences en acides aminés des récepteurs P2Y humains authentiques à l'aide de l'algorithme ClustalX. L'arbre inclut quelques séquences de récepteurs orphelins structurellement proches, qui pourraient ultérieurement venir compléter la famille P2Y. Les couleurs identifient les deux sous-groupes structurellement distincts de récepteurs P2y. 
sont libérés dans la circulation par les cellules lésées - globules rouges et cellules endothéliales - et plus spécifiquement à partir des plaquettes activées où ils sont stockés à très forte concentration dans les granules denses [10]. On comprend que l'identification des récepteurs responsables des effets de l'ADP sur les plaquettes sanguines était d'un intérêt considérable pour la mise au point de médicaments anti-plaquettaires à visée anti-thrombotique.

Deux récepteurs, $\mathrm{P} 2 \mathrm{y}_{1}$ et $\mathrm{P} 2 \mathrm{y}_{12}$, sont responsables simultanément de l'activation et de l'agrégation des plaquettes sanguines par l'ADP (Figure 3). L'activation du récepteur $P 2 y_{1}$, couplé à $G \alpha_{q}$, induit la mobilisation des stocks internes de calcium, le changement de forme des plaquettes, qui de discoïdes deviennent sphériques et émettent des pseudopodes, et le déclenchement de l'agrégation plaquettaire, tandis que l'activation du récepteur $\mathrm{P} 2 \mathrm{Y}_{12}$, couplé à $G \alpha_{i 2}$, complète la réponse plaquettaire, amplifie l'agrégation et permet son maintien selon un mécanisme qui implique la PI-3 kinase $\gamma[11]$. Chacun des deux récepteurs est nécessaire pour une agrégation normale en réponse à I'ADP. L'inhibition de l'un ou l'autre par des agents pharmacologiques ou par invalidation du gène, se traduit par une absence d'agrégation plaquettaire en réponse à la stimulation par l'ADP ainsi que par l'inhibition de l'agrémation induite par d'autres agents agrégants tels que le collagène ou la thrombine. Cependant, étant donnée la spécificité fonctionnelle de chacun de ces récepteurs, selon que l'on touche au récepteur $P 2 y_{1}$ ou bien au récepteur $P 2 y_{12}$, les réponses ne sont pas affectées de la même façon. Ainsi, des études chez des souris déficientes pour le récepteur $\mathrm{P} 2 \mathrm{Y}_{1}[12,13]$ ou bien l'emploi d'antagonistes sélectifs du récepteur $\mathrm{P} 2 \mathrm{y}_{1}$ montrent que le changement de forme et l'agrégation plaquettaire induits par l'ADP sont complètement inhibés,

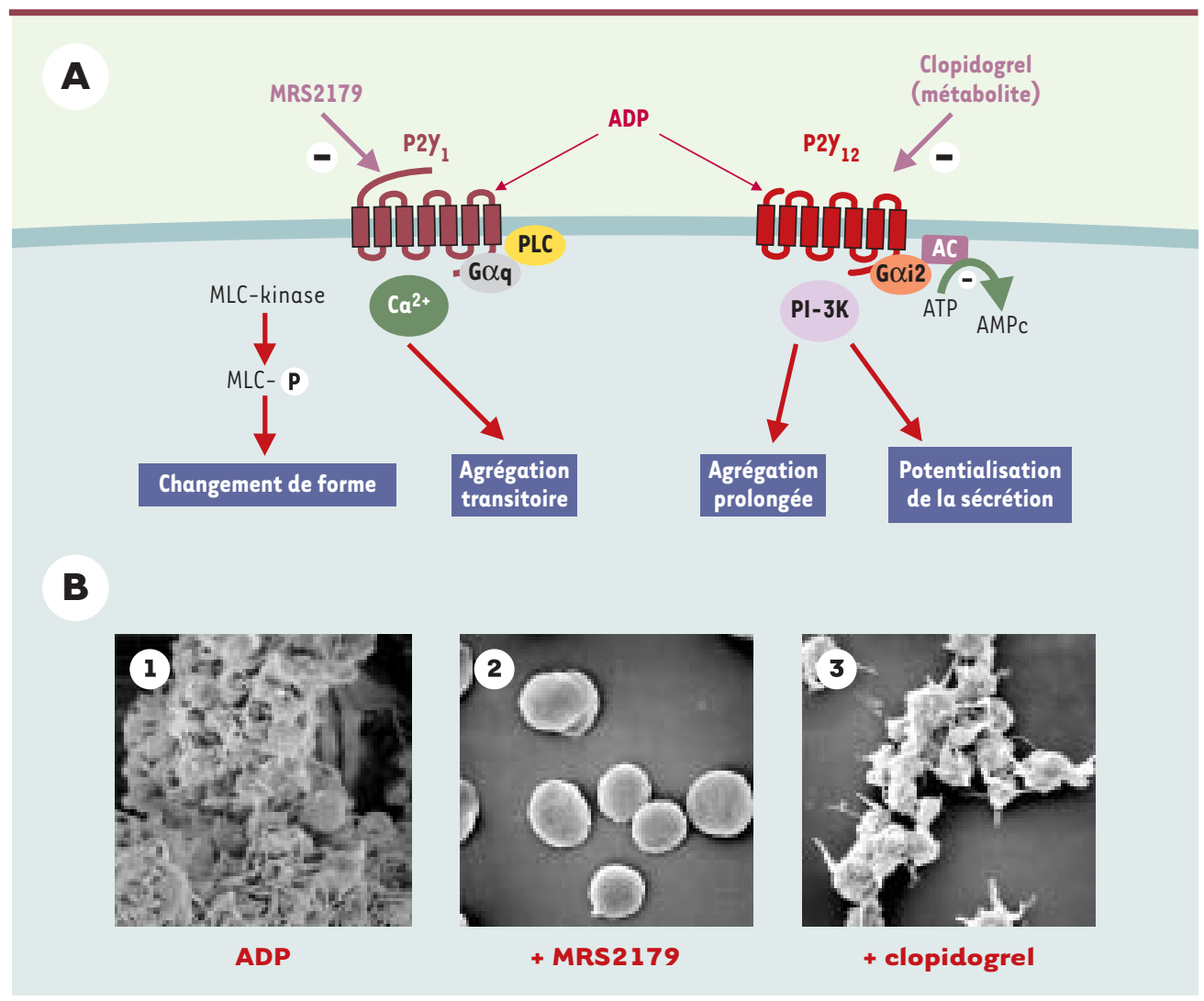

Figure 3. Rôle des récepteurs $\mathbf{P 2} \mathrm{Y}_{1}$ et $\mathbf{P} 2 \mathrm{Y}_{12}$ dans l'agrégation plaquettaire. $A$. Le récepteur $\mathrm{P} 2 \mathrm{Y}_{1}$ est responsable de la mobilisation des stocks internes de calcium, du changement de forme des plaquettes et d'une agrégation transitoire lors de la stimulation par l'ADP. Le récepteur P2 $_{12}$, couplé à l'inhibition de l'adénylyl cyclase (AC) via $G \alpha_{i 2}$ et à l'activation de la PI-3 kinase est responsable de l'amplification des réponses et de la stabilisation de l'agrégation. Il est la cible moléculaire des médicaments antithrombotiques comme le clopidogrel. Chacun des deux récepteurs est nécessaire pour une réponse normale des plaquettes à l'ADP et à des agents comme le collagène ou la thrombine. B. Aspects morphologiques. L'agrégation plaquettaire induite par l'ADP (1) est inhibée sélectivement par un antagoniste du récepteur $\mathrm{P}_{2} \mathrm{Y}_{1}$ (2) ou par un inhibiteur du récepteur $\mathrm{P} 2 \mathrm{Y}_{12}$ (3). Dans ce dernier cas, le changement de forme des plaquettes n'est pas affecté. tandis que l'agrégation induite par le collagène n'est que partiellement réduite. En revanche, chez les souris déficientes pour le récepteur $\mathrm{P} 2 \mathrm{y}_{12}$ ou bien lors d'un traitement par des antagonistes sélectifs du récepteur P2y ${ }_{12}$, l'agrégation plaquettaire est inhibée, mais le changement de forme persiste $[10,14]$. L'activation par d'autres agonistes est très affectée par le blocage des récepteurs $P 2 y_{12}$, soulignant le rôle clé de ce récepteur dans l'amplification des réponses plaquettaires à tous les stimulus. Des mutations ponctuelles et des délétions dans le gène codant pour ce récepteur ont été identifiées chez des patients atteints d'un syndrome hémorragique modéré qu'explique l'inhibition sélective de l'agrégation plaquettaire à I'ADP [10]. Ces propriétés font de ce récepteur une cible de choix pour des médicaments antithrombotiques. Ainsi, le récepteur P2y 12 est la cible moléculaire d'une classe 
de médicaments déjà sur le marché, les thiénopyridines anti-agrégantes, ticlopidine (Ticlid ${ }^{\circledR}$ ) et clopidogrel (Plavix $^{\circledR}$ ), qui agissent in vivo via un métabolite actif [15]. Le clopidogrel en particulier a prouvé son efficacité dans la prévention secondaire des accidents ischémiques dans de très grands essais cliniques multicentriques (CAPRIE, (URE), et est aujourd'hui de prescription courante. Des analogues de I'ATP, antagonistes sélectifs du récepteur P2 $y_{12}$, ont également été proposés et sont actuellement testés chez l'homme [16]. Depuis le clonage du récepteur P2 ${ }_{12}$, de nouvelles molécules sont apparues qui sont en cours d'évaluation préclinique. Concernant le récepteur $P 2 Y_{1}$, des études sont en cours pour évaluer son intérêt comme cible de nouveaux antithrombotiques. En effet, on a montré une diminution significative de la mortalité chez les souris déficientes pour l'expression du récepteur $\mathrm{P} 2 \mathrm{y}_{1}$, ainsi que chez des souris sauvages traitées par des antagonistes sélectifs du récepteur P2 ${ }_{1}$, lors de l'induction d'un processus thrombotique généralisé par injection d'un mélange de collagène et d'adrénaline ou bien de facteur tissulaire [13, 17]. Des études complémentaires dans des modèles de thrombose localisée sont en cours et confirment les études préliminaires [18]. Un important effort en pharmacochimie est souhaitable afin de disposer d'outils plus performants en termes de sélectivité et d'efficacité. Quoiqu'il en soit, on peut espérer que soient développés dans un avenir proche de nouveaux antithrombotiques dirigés contre les récepteurs P2 des plaquettes.

\section{Hydratation du mucus}

L' « escalator » mucociliaire est un mécanisme vital de protection des poumons. II intègre trois éléments : la sécrétion de mucus par les cellules caliciformes et les cel- lules des glandes sous-muqueuses pour capturer les particules étrangères, les battements ciliaires pour propulser ce tapis muqueux et le transport épithélial d'ions et d'eau pour maintenir sa fluidité [19]. L'ATP et I'UTP activent ces trois composantes. En particulier, ils stimulent la sécrétion apicale de chlorure et d'eau par les cellules épithéliales des voies respiratoires, en déclenchant l'ouverture des canaux chlorure à rectification sortante (ORCC) sensibles au $\mathrm{Ca}^{2+}$ (Figure 4). L'invalidation génique chez la souris a permis d'établir que c'est le récepteur $\mathrm{P}_{2} \mathrm{y}_{2}$ qui joue un rôle déterminant dans cette stimulation [20]. En effet, l'action de l'ATP et de I'UTP sur le transport de $\mathrm{Cl}^{-}$à travers la muqueuse trachéale a été complètement abolie chez les souris $P 2 y_{2}{ }^{-/-}$, qui gardent par ailleurs un phénotype globalement normal. Ce mécanisme, indépendant du CFTR (cystic fibrosis transmembrane regulator), est opérant dans des cellules épithéliales provenant de patients atteints de mucoviscidose. Dès lors, l'administration de nucléotides en aérosol apparaît comme un traitement symptomatique potentiel de maladies dans lesquelles les voies aériennes sont obstruées par un mucus visqueux, ce qui est le cas dans la mucoviscidose ou dans la bronchite chronique. Étant donné que l'adénosine, produit de dégradation de I'ATP, exerce une action bronchoconstrictrice, I'UTP s'est imposé comme le meilleur candidat. Des essais de phase I chez des volontaires ont montré que I'UTP en aérosol est bien toléré et accélère la clairance mucociliaire mesurée à l'aide d'une méthode scintigraphique [19]. Chez des patients souffrant de mucoviscidose, la combinaison d'UTP (qui stimule la sécrétion épithéliale de chlorure) et d'amiloride (qui inhibe la réabsorption épithéliale de sodium) a rétabli des valeurs de clairance mucociliaire proches de la normale. La dégradation rapide des nucléotides par les ecto-nucléotidases est un handi-

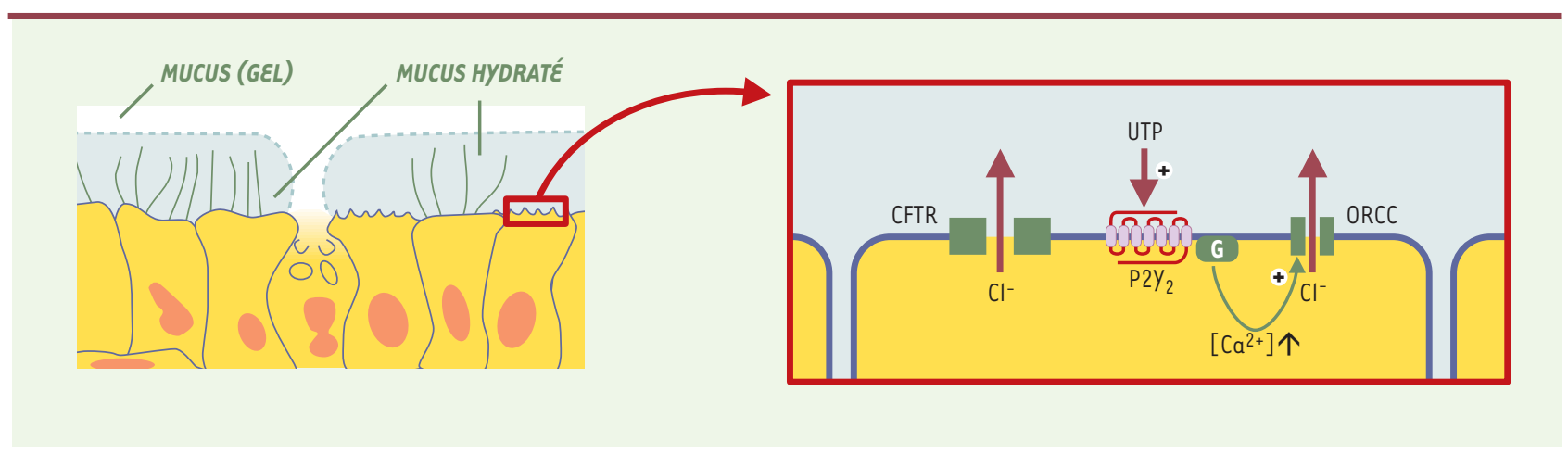

Figure 4. Rôle du récepteur P2y ${ }_{2}$ dans le contrôle de l" « escalator » mucociliaire. La partie gauche schématise côte à côte les trois composantes de l'escalator mucociliaire : cellule caliciforme sécrétrice de mucus, cellule sécrétrice d'électrolytes et d'eau que fluidifient le mucus et cellule ciliaire assurant la propulsion du mucus. La partie droite est un agrandissement d'une cellule sécrétrice d'électrolytes et d'eau. Elle montre que la sécrétion apicale de $\mathrm{Cl}^{-}$fait intervenir deux transporteurs : le CFTR et les canaux à rectification sortante (ORCC). L'activation du récepteur P2Y 2 par l'UTP induit l'ouverture des canaux ORCC via une augmentation de $\mathrm{Ca}^{2+}$ cytosolique. 
cap qui limite leur utilisation thérapeutique. C'est pourquoi, dans un essai clinique de phase II actuellement en cours pour le traitement de la bronchite chronique, c'est un dérivé dinucléotidique de I'UTP à durée de vie prolongée, I'U $p_{4} U$ (INS365), qui est testé. La stimulation du récepteur $\mathrm{P} 2 \mathrm{Y}_{2}$ de l'épithélium conjonctival augmente également la sécrétion d'électrolytes et d'eau, ce qui pourrait améliorer la lubrification de la cornée [21]. L'Up $p_{4} U$ en collyre fait actuellement l'objet d'un essai de phase III pour le traitement du syndrome de l'œil sec, une affection fréquente dans laquelle le déficit de production des larmes entraîne une irritation oculaire, voire des lésions de la cornée.

\section{Hématopoïèse et système immunitaire}

Les cellules de la lignée HL-60 proviennent d'un patient atteint de leucémie aiguë promyélocytaire et ont été abondamment utilisées pour l'étude de la maturation granulocytaire. En effet, différents agents (DMSO, acide rétinoïque, G-CSF) induisent leur différenciation en cellules présentant certaines propriétés fonctionnelles de neutrophiles, tandis que d'autres agents comme la vitamine $D_{3}$ les différencient en monocytes. Le clonage du récepteur P2 $\mathrm{Y}_{11}$ a été quasi simultané à l'observation que I'ATP induisait la différenciation granulocytaire des cellules $\mathrm{HL}$ 60 via une augmentation d'AMP cyclique [22]. Cet effet de I'ATP est reproduit par les agonistes du récepteur $P 2 Y_{11}$. L'expression d'ARN messagers P2 $y_{11}$ est détectable dans les cellules HL-60 et augmente en réponse aux agents qui induisent leur différenciation en granulocytes. Ces résul-

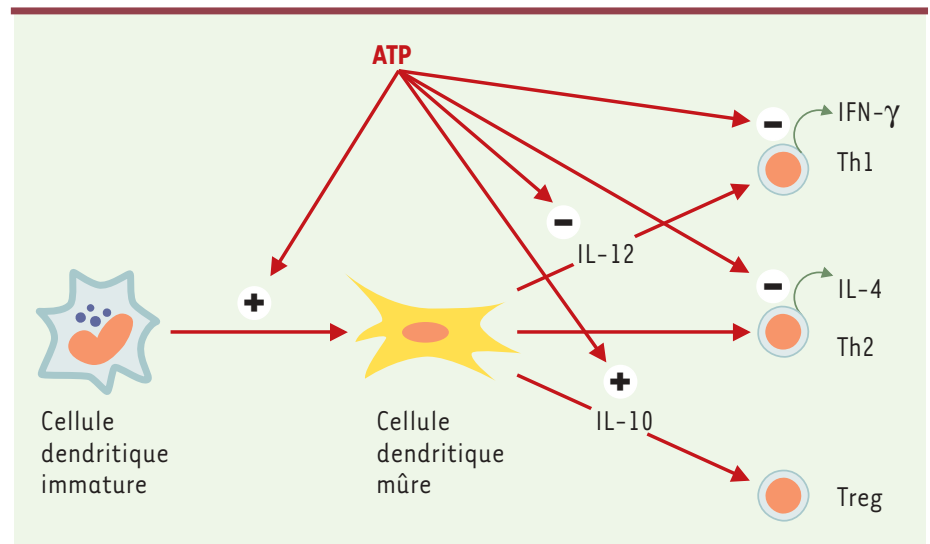

Figure 5. Action immunomodulatrice de l'ATP. L'ATP induit la maturation des cellules dendritiques, inhibe leur sécrétion d'IL-12 et potentialise celle d'IL-10. Par ailleurs, il agit directement sur les cellules T CD4 $4^{+}$en inhibant leur prolifération et la sécrétion des cytokines tant Thl (IFN- $\gamma$ ) que Th2 (IL-4). L'effet sur les cellules T régulatrices (Treg) est hypothétique. Ces actions sont liées à une augmentation d'AMP cyclique et, dans les cellules dendritiques, impliquent le récepteur $\mathrm{P} 2 \mathrm{Y}_{11}$. tats suggèrent que des agonistes du récepteur $\mathrm{P} 2 \mathrm{y}_{11}$ pourraient traiter certaines leucémies en induisant une différenciation cellulaire, concept dont la validité est démontrée par l'efficacité de l'acide rétinoïque dans le traitement de la leucémie aiguë promyélocytaire $(\rightarrow)$. Malgré une littérature assez abondante, il était difficile jusqu'à il y a peu d'intégrer en un ensemble cohérent les actions des nucléotides sur les cellules du système immunitaire. Des résultats récents montrent que l'ATP agit sur les cellules dendritiques et les lymphocytes T CD4 $4^{+}$(Figure 5), via une augmentation d'AMP cyclique, les agonistes du récepteur $\mathrm{P}_{2} \mathrm{Y}_{11}$ induisent la maturation des cellules dendritiques, inhibent leur production d'IL-12 et potentialisent celle d'IL-10 [23, 24]. Dès lors, ces agonistes vont orienter les cellules $T C D 4^{+}$vers une réponse Th2 plutôt que Thl, ou favoriser le développement de cellules $T$ régulatrices (Treg) associées à la tolérance immunitaire. Parallèlement, l'action directe de l'ATP sur les lymphocytes T CD4 ${ }^{+}$conduit, également via l'AMP cyclique, à une inhibition de leur prolifération et de la sécrétion de cytokines en réponse à l'engagement du TCR (T cell receptor) [25]. Cette action coordonnée est réminiscente de celle de la prostaglandine $\varepsilon_{2}$, également libérée par les cellules «stressées », et inhibe les lymphocytes $T$ via le récepteur de la prostaglandine $\varepsilon 2$ et les cellules présentatrices d'antigène via le récepteur de la prostaglandine $\varepsilon 4$ [26]. Vu sa faible affinité pour son ligand naturel, le récepteur P2y répondra sélectivement à I'ATP libéré en grandes quantités par les cellules nécrotiques, ou concentré dans l'espace intercellulaire restreint de la synapse formée entre cellules dendritiques et cellules T. Ces observations peuvent conduire au développement de nouveaux agents anti-inflammatoires et immunosuppresseurs. D'autres récepteurs P2y paraissent impliqués dans le système immunitaire, en particulier le récepteur P2 $y_{6}$, qui est exprimé dans les lymphocytes $T C D 4^{+}$et $\mathrm{CD}^{+}$activés, notamment ceux qui infiltrent l'intestin de patients atteints d'iléite de Crohn [27]. Par ailleurs, la production d'IL-8 par les monocytes en réponse au lipopolysaccharide est due en partie à une activation autocrine du récepteur ${\mathrm{P} 2 \mathrm{Y}_{6}}_{6}$ [28].

\section{Conclusions}

On le voit, depuis le premier clonage d'un récepteur P2Y en 1993, de nombreux sous-types ont été identifiés et leurs rôles dans les grands systèmes, les tissus et les cellules sont de mieux en mieux caractérisés. Les espoirs thérapeutiques suscités dans les domaines décrits dans cet article sont un encouragement à poursuivre les efforts dans cette voie et à étendre le champ d'investigation à tous les domaines dans lesquels les nucléotides extracellulaires ont une action physiologique par eux-mêmes ou comme agents potentialisateurs d'hormones, de neurotransmetteurs, de cytokines ou d'autres médiateurs. $\diamond$ 


\section{SUMMARY}

P2y receptors of extracellular nucleotides:

from cloning to physiology

The appearance of adenine and uracil nucleotides in the extracellular fluids can result from cell necrosis, exocytosis of secretory granules (synaptic vesicles, platelet dense bodies) or efflux through membrane transport proteins, the identity of which remains controversial. Extracellular nucleotides are rapidly degraded by ectonucleotidases ubiquitously present on the plasma membrane. The actions of extracellular nucleotides are mediated by two families of receptors : the $P 2 X$ receptors that are ligand-gated ion channels and the P2Y receptors that are coupled to $G$ proteins. So far eight genuine human P2y receptors have been identified. According to sequence homology they belong to two distinct subgroups. On the one hand the $\mathrm{P} 2 \mathrm{y}_{1}, \mathrm{P} 2 \mathrm{y}_{2}, \mathrm{P} 2 \mathrm{y}_{4}$, $P 2 y_{6}$ and $P 2 y_{11}$ receptors are all coupled to phospholipase $C$ via a $G \alpha_{q / 11}$ protein, while the $P 2 Y_{11}$ receptor is additionnally coupled to $G \alpha_{s}$ and adenylyl cyclase stimulation. On the other hand, the P2 $\mathrm{y}_{12}, \mathrm{P}_{2} \mathrm{y}_{13}$ and $\mathrm{P} 2 \mathrm{y}_{14}$ receptors are coupled to $G_{i}$. The physiopathological roles and pharmacotherapeutic potential of several P2y receptors have now been established. Platelet aggregation by ADP involves the dual activation of P2y and $P 2 y_{12}$ receptors and the $P 2 y_{12}$ receptor is the target of the antithrombotic agent clopidogrel. Via $\mathrm{P} 2 \mathrm{y}_{2}$ receptors, nucleotides stimulate chloride secretion by epithelial cells and thereby promote the hydration of airway mucus and the lubrification of the ocular surface. Clinical trials of uracil nucleotides in sprays or eye drops are currently underway for the treatment of chronic bronchitis and dry eye syndrome. Finally, recent results suggest a role for the $\mathrm{P} 2 \mathrm{Y}_{11}$ receptor in granulocyte differentiation and immunomodulation via effects on both dendritic cells and $C D 4^{+} T$ cells. $\Delta$

\section{RÉFÉRENCES}

1. Ralevic V, Burnstock $G$. Receptors for purines and pyrimidines. Pharmacol Rev $1998 ; 50: 413-92$.

2. Burnstock G. Purinemediated signalling in pain and visceral perception. Trends Pharmacol Sci 2001 ; 22 : 182-8.

3. Schneider SW, Egan ME, Jena BP, Guggino WB, Oberleithner $\mathrm{H}$, Geibel JP. Continuous detection of extracellular ATP on living cells by using atomic force microscopy.
Proc Natl Acad Sci USA 1999 ; 96 : 12180-5.

4. Watt WC, Lazarowski $\varepsilon R$, Boucher RC. Cystic fibrosis transmembrane regulatorindependent release of ATP. Biol Chem 1998 ; 273 . 14053-8.

5. Zimmermann H. Extracellular metabolism of ATP and other nucleotides. NaunynSchmiedeberg's Arch Pharmacol 2000 ; 362 : 299-309.

6. Marcus AJ, Broekman MJ, Drosopoulos JH, et al. The endothelial cell ecto-
ADPase responsible for inhibition of platelet function is CD39. J Clin Invest 1997 ; 99 : 1351-60.

7. Enjyoji K, Sevigny J, Lin Y, et al. Targeted disruption of CD39/ATP

diphosphohydrolase results in disordered hemostasis and thromboregulation. Nat Med 1999 ; 5 :1010-7.

8. Leurs $R$, Watanabe $T$, Timmerman $\mathrm{H}$. Histamine receptors are finally coming out. Trends Pharmacol Sci 2001 ; 22 : 337-9.

9. Gaarder A, Jonsen L, Laland S, Hellem A, Owren PA. Adenosine diphosphate in red cells as a factor in the adhesiveness of human blood platelets. Nature 1961 ; 192 : 531-2.

10. Gachet C. ADP receptors of platelets and their inhibition. Thromb Haemost 2001 ; 86 : 222-32.

11. Trumel C, Payrastre B, Plantavid $M$, et al. A key role of adenosine diphosphate in the irreversible platelet aggregation induced by the PARl-activating peptide through the late activation of phosphoinositide 3 kinase. Blood 1999 ; 94 : 4156-65.

12. Fabre JE, Nguyen M, Latour $A$, et al. Decreased platelet aggregation, increased bleeding time and resistance to thromboembolism in P2y ${ }_{1}$-deficient mice. Nat Med 1999; 5 : 1199-202.

13. Leon C, Hechler B, Freund M, et al. Defective platelet aggregation and increased resistance to thrombosis in purinergic $\mathrm{P} 2 \mathrm{Y}$ (1) receptornull mice. J Clin Invest 1999 ; 104 : 1731-7.

14. Foster CJ, Prosser DM, Agans JM, et al. Molecular identification and characterization of the platelet ADP receptor targeted by thienopyridine antithrombotic drugs. J Clin Invest 2001 ; 107 : 1591-8.

15. Savi P, Pereillo JM, Uzabiaga MF, et al. Identification and biological activity of the active metabolite of clopidogrel. Thromb
Haemost 2000 ; 84 : 891-6.

16. Humphries RG.

Pharmacology of AR-

C69931MX and related

compounds: from

pharmacological tools to clinical trials.

Haematologica 2000 ; 85 : 66-72.

17. Leon $C$, Freund $M$, Ravanat $C$, Baurand A, Cazenave JP, Gachet $C$. Key role of the $\mathrm{P} 2 \mathrm{Y}(1)$ receptor in tissue factor-induced thrombindependent acute thromboembolism: studies in P2y(1)-knockout mice and mice treated with a P2y (1) antagonist. Circulation $2001 ; 103: 718-23$

18. Lenain N, Freund M, Léon C, Cazenave JP, Gachet C. Reduced platelet thrombus formation in arterioles of P2y ${ }_{1}$ receptor-deficient mice. Thromb Haemost 2001 ; 86 (suppl) : P1926 (abstract).

19. Olivier KN, Bennett WD, Hohneker KW, et al. Acute safety and effects on mucociliary clearance of aerosolized uridine 5 'triphosphate \pm amiloride in normal human adults. Am J Respir Crit Care Med 1996 ; $154:$ 217-23.

20. Cressman VL, Lazarowski $\varepsilon$, Homolya L, Boucher RC, Koller BH, Grubb BR. Effect of loss of $\mathrm{P} 2 \mathrm{Y}_{2}$ receptor gene expression on nucleotide regulation of murine epithelial $\mathrm{Cl}^{-}$transport. J Biol Chem 1999 ; 274 : 26461-8.

21. Li Y, Kuang K, Wen Q, Rosskothen H, Fiscbarg J. Rabbit conjunctival epithelium transports fluid and $\mathrm{P} 2 \mathrm{Y}_{2}$ receptor agonists stimulate $\mathrm{Cl}^{-}$and fluid secretion. Am J Physiol 2001 ; 281 : C595-602.

22. Jiang $L$, Foster FM, Ward $P$, Tasevski V, Luttrell BM, Conigrave AD. Extracellular ATP triggers cyclic AMPdependent differentiation of HL-60 cells. Biochem Biophys Res Commun 1997 ; 232 : 626-30.

23. la Sala A, Ferrari D, Corinti S, Cavani A, Di Virgilio F, Girolomoni G. Extracellular ATP induces a distorted 
maturation of dendritic cells and inhibits their capacity to initiate Thl responses. J

Immunol 2001 ; 166 :1611-7.

24. Wilkin F, Duhant $X$, Bruyns $C$, Suarez-Huerta N, Boeynaems JM, Robaye B. The P2 ${ }_{11}$ receptor mediates the ATP-induced maturation of human monocyte-derived dendritic cells. J Immunol $2001 ; 166: 7172-7$.

25. Duhant $X$, Schandené $L$, Bruyns C, et al. Extracellular adenine nucleotides inhibit the activation of human $\mathrm{CD}^{+}$T lymphocytes. J Immunol 2002 ; 169 : 15-21.

26. Nataraj C, Thomas DW, Tilley $\mathrm{SL}$, et al. Receptors for prostaglandin $\varepsilon_{2}$ that regulate cellular immune responses in the mouse. J Clin Invest 2001 ; 108 : 1229-35.

27. Somers GR, Hammet FMA, Trute L, Southey MC, Venter DJ. Expression of the $\mathrm{P}_{2} \mathrm{y}_{6}$ purinergic receptor in human T cells infiltrating inflammatory bowel disease. Lab Invest 1998 ; 78 : 1375-83.

28. Warny M, Aboudola $S$, Robson SC, et al. P2y nucleotide receptor mediates monocyte interleukin-8 production in response to UDP or lipopolysaccharide. J Biol Chem 2001 ; 276 : 26051-6.

29. Léon C, Vial C, Cazenave JP, Gachet C. Cloning and sequencing of a human CDNA encoding endothelial P2y receptor. Gene 1996 ; 171 295-7.

30. Parr CE, Sullivan DM, Paradiso AM, et al. Cloning and expression of a human $P_{2 U}$ nucleotide receptor, $a$ target for cystic fibrosis pharmacotherapy. Proc Nat Acad Sci USA 1994 ; 91 : 3275-9

31. Communi D, Pirotton S,
Parmentier M, Boeynaems JM. Cloning and functiona expression of a human uridine nucleotide receptor. J Biol Chem 1995 ; 270 : 30849-52.

32. Communi D, Parmentier M Boeynaems JM. Cloning, functional expression and tissue distribution of the

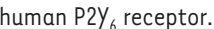
Biochem Biophys Res Commun 1996; 222 : 303-8.

33. Communi $D$, Govaerts $C$, Parmentier M, Boeynaems JM. Cloning of a human purinergic receptor coupled to phospholipase $\mathrm{C}$ and adenylyl cyclase. J Biol Chem 1997 ; 272 : 31969-73.

34. Hollopeter G, Jantzen HM, Vincent $D$, et al. Identification of the platelet ADP receptor targeted by antithrombotic drugs. Nature 2001 ; 409 : 202-6.

35. Zhang FL, Luo L, Gustafson $\varepsilon$, et al. ADP is the cognate ligand for the orphan $\mathrm{G}$ protein coupled receptor SP1999. J Biol Chem 2001 $276: 8608-15$.

36. Communi D, Suarez Gonzalez $\mathrm{N}$, Detheux M, et al. Identification of a novel human ADP receptor coupled to $\mathrm{G}_{\mathrm{i}}$. J Biol Chem 2001 $276: 41479-85$

37. Chambers JK, Macdonald LE, Sarau HM, et al. A Gprotein-coupled receptor for UDP-glucose. J Biol Chem $2000 ; 275$ :

10767-71.

\section{TIRÉS À PART}

J.M. Boeynaems et C. Gachet 